\title{
LOS USOS DEL AFECTO EN EL CURRÍCULUM ESCOLAR*
}

\author{
Claudia Matus"* \\ Pontificia Universidad Católica de Chile (UC), Santiago - Región Metropolitana, Chile
}

RESUMEN: En este artículo exploramos como el currículum escolar usa el afecto, entendido como una tecnología o fuerza para incitar ciertos comportamientos y moralidades, en estudiantes en Chile. En la medida en que el currículum organiza el conocimiento en distintas dimensiones, como por ejemplo las actitudes, es que el afecto consolida la neutralidad requerida para mantener órdenes sociales y culturales; particularmente cuando nos referimos a categorías de raza, etnia, clase social, género y sexualidad. Por lo tanto, el objetivo es mostrar como el afecto es usado a través del currículum escolar para promover formas neutrales de producir sujetos escolares. Más que dar respuestas, el esfuerzo de este artículo está en producir problemáticas acerca de cómo se ha sofisticado la regulación de los sujetos a través del currículum. Palabras clave: Currículum como conocimiento. Afecto. Producción de sujeto.

\section{USOS DO AFETO NO CURRÍCULO ESCOLAR}

RESUMO: Neste artigo, exploramos como o currículo escolar usa o afeto, entendido como uma tecnologia ou força para incitar certos comportamentos e moralidades, em estudantes no Chile. À medida que o currículo organiza o conhecimento em distintas dimensões, por exemplo, as atitudes, é que o afeto consolida a neutralidade requerida para manter ordens sociais e culturais, particularmente quando nos referimos a categorias de raça, etnia, classe social, gênero e sexualidade. Portanto, o objetivo é mostrar como o afeto é usado por meio do currículo escolar para promover formas neutras de produzir sujeitos escolares. Mais que dar respostas, o esforço deste artigo está em produzir uma problemática sobre como a regulação dos sujeitos foi se sofisticando através do currículo.

Palavras-chave: Currículo como conhecimento. Afeto. Produção de sujeito.

http://dx.doi.org/10.1590/0102-4698153449

"La escritura de este artículo fue auspiciado por el fondo VRI Puente de la Pontificia Universidad Católica de Chile, Proyeto \#3114200. Las ideas expresadas en este artículo son de responsabilidad de la autora y no necesariamente reflejan aquellas de la institución que otorgó los recursos.

"* Ph.D en Educación, Universidad de Illinois, Estados Unidos. Profesora Asociada de la Facultad de Educación Pontificia Universidad Católica de Chile. E-mail: < cmatusc@uc.cl>. 


\section{USES OF AFFECT IN SCHOOL CURRÍCULUM}

ABSTRACT: In this paper, we explore the ways school curriculum uses affection to incite specific behaviors and moralities in students. As the curriculum organizes knowledge through different dimensions, for example in attitudes, is that affection that consolidates the required neutrality to sustain social and cultural orders; particularly when we are referring to the categories race, ethnicity, social class, gender, and sexuality. Thus, the goal is to show how affection is used in the school curriculum to promote neutral ways to produce school subjects. More than providing specific answers, the purpose of this article is to produce a problem on the ways how sophisticated mechanisms to regulate subjects have been set up.

Keywords: Curriculum as knowledge. Affect. Producing subjects.

\section{PRESENTACIÓN}

En el contexto de las reformas en educación en Chile se ha planteado como elemento vector la renovación del relato de la inclusión en las políticas emanadas desde el Ministerio de Educación. En este sentido, se ha promulgado recientemente la Ley de Inclusión Escolar que se sostiene en dos orientaciones clave: (a) el principio de no discriminación arbitraria e inclusión y (b) el principio de gratuidad progresiva. Esta Ley entra en vigencia desde el 1 de marzo de 2016. En términos específicos esta ley modifica cuatro cuerpos legales que operan actualmente en la legislación chilena: La Ley General de Educación (en donde se prohibirá la petición de antecedentes socioeconómicos, de escolaridad, estado civil, de patrimonio de la familia a la hora de postular a los establecimientos escolares); la Ley de Subvenciones (en donde se regulará que todos los recursos que reciba un sostenedor deberán ser destinados únicamente para fines educativos y además instalará una normativa en donde son los apoderados los que escogen los establecimientos donde quieren que sus hijos e hijas estudien); la Ley de Aseguramiento de la Calidad (que ampliará las atribuciones de la Superintendencia de Educación Escolar); y la Ley de Subvención Escolar Preferencial (que expandirá el rango de valoración para definir a los y las estudiantes preferenciales para otorgar subvención). De esta manera lo que tendremos como resultado de la aplicación de esta ley es que los establecimientos escolares serán más heterogéneos y en donde las valoraciones culturales y sociales que se hacen actualmente de los y las estudiantes estarán, de alguna manera, siendo más evidentes. 
Junto a este escenario, a nivel nacional operan otras leyes que están configurando nuevos escenarios para pensar los espacios escolares. Me refiero a la Ley de Anti Discriminación promulgada en el año 2012, que instala un mecanismo judicial para penalizar situaciones de discriminación arbitraria; y el Acuerdo de Unión Civil, que pone término a la discriminación y desprotección de parejas que conviven y que son de igual o distinto sexo. Ambas leyes lo que hacen, de múltiples maneras, es ir reconfigurando espacios de acción cultural en la sociedad civil que hace imperativo que nos detengamos a pensar otras formas de organizar la política en educación.

En este sentido quiero proponer como el currículum escolar, entendido como conocimiento, debiera someterse a la pregunta de cómo aporta a la producción de ciudadanos y ciudadanas con capacidades para poder integrar estos nuevos espacios sociales y culturales. En el caso puntual de aquellas políticas curriculares que se enmarcan dentro de las políticas de diversidad, al solo enfatizar dimensiones administrativas y pedagógicas para hacerse cargo de conceptualizaciones particulares sobre diversidad, promueven que tanto profesores/as como estudiantes asuman posturas reduccionistas y esencialistas acerca de las "diferencias," aún cuando busquen contrarrestar los efectos opresivos de éstas. La narrativa que subyace a estas políticas tiene que ver con que los individuos son distintos, ya sea por raza, género, sexualidad, clase social, capacidad, edad, etc., y luego de aceptar este hecho, los y las profesoras tienen que plantear como medir, monitorear y controlar este "fenómeno" (BAEZ, 2004). Es así en la Política Nacional de Educación Especial en Chile del año 2005, por ejemplo, donde se plantea lo siguiente,

[...] con frecuencia, los estudiantes que provienen de sectores sociales más desfavorecidos o de otras culturas, que tienen una menor vinculación con los objetivos de la escuela, suelen presentar dificultades de aprendizaje y requieren, por tanto recursos adicionales para participar y aprender junto a sus compañeros [...] (Política Nacional de Educación Especial, 2005, p. 35).

Desde aquíse desprenden varios supuestos que son importantes de problematizar. Primero, hay una reducción, normalización y esencialización de grupos de estudiantes que son confinados a grupos "reconocibles" tales como aquellos que provienen de "sectores sociales desfavorecidos" y que pertenecen a "otras culturas." Además, se plantea de manera abierta que existen "rasgos" o "atributos" que exhiben "estos estudiantes" y que serán traducidos como "dificultades de aprendizajes" a través de los diagnósticos utilizados. Más importante aún, estas supuestas dificultades de aprendizaje "son 
reales" por el hecho de que estos/estas estudiantes "vienen de estos lugares" que por obviedad están "menos vinculados con los objetivos de la escuela." De manera casi unívoca una pensaría que estas formas de redactar las políticas educativas tienen serios problemas de, al menos, sesgo y que, así como están redactadas, más que provocar un cambio sustantivo, instalan lógicas de discriminación evidentes.

Estas formas de pensar la diferencia son las que me motivan a problematizar las ideas que operan tras las formas de abordar temas de diversidad en los currículos escolares. La neutralidad que se instala a partir de las formas de pensar a los sujetos distintos constituye una de las grandes barreras para efectivamente poder avanzar en unos currículos y prácticas pedagógicas más críticas para abordar temas como la discriminación, la desigualdad y la injusticia social. Las formas de organizar nuestras experiencias desde el binomio nosotros/ellos (e.g. nacional/extranjero); las correspondencias inapelables entre espacios e identidades (e.g. los peruanos que vienen de Perú deben exhibir su "peruanidad"); los esencialismos y el sentido común, etc. son aprendidas y el currículum se transforma en un instrumento significativo para su reproducción. Dicho de otra manera, el currículum inventa la trayectoria de negación del otro, que luego él que narra su marginalidad lo relata como su "experiencia de marginación o discriminación," sin embargo, lo que narra no es más que la narración de la invención de esta marginación (BRITZMAN, 1992).

En este sentido, me gustaría problematizar la forma en que el currículum en Chile ha incluido nuevos mecanismos para solidificar formas a-problemáticas de trabajar las relaciones de poder que producen contextos de discriminación.

Para producir esta problemática primero presentaré como se organizan tres elementos relevantes para este ejercicio: lo curricular, lo discursivo y lo generativo. Luego, usaré algunas cifras que tienen que ver con temas de discriminación en Chile para indicar algunos de los órdenes sociales y culturales que circulan en la imaginería de las personas encuestadas. Más tarde, presentaré algunas políticas curriculares que han incluido temas como los objetivos actitudinales de manera relevante para la consolidación de los aprendizajes esperados. Desde aquí hago un análisis de cómo se usa el afecto para la consolidación de la neutralidad de los abordajes de temas relacionados con discriminación e injusticias sociales. Más que dar respuestas, el esfuerzo de este artículo está en producir unas problemáticas acerca de cómo se ha ido sofisticando la regulación de los sujetos a través del currículum. 


\section{LO CURRICULAR, DISCURSIVO Y GENERATIVO}

Para poder problematizar las experiencias de aquellos que son definidos o enseñados como "distintos" en los contextos escolares, es necesario entender el currículum como un texto vivido (PINAR; REYNOLDS; SLATTERY; TAUBMAN, 2000; AOKI, 1993; HUEBNER, 1975; JARDINE, 1992). En otras palabras, las experiencias de marginalización y de normalidad están disponibles en las trayectorias curriculares de manera tal que son aprehendidas y relatadas por los y las estudiantes como las "experiencias de marginación y de privilegio." Por lo tanto, podemos pensar que el currículum actúa como un texto generativo. Generativo de ideas totalizantes habilitadas en las "experiencias curriculares", en donde las contradicciones de cómo se producen ciertos tipos de conocimientos son identificables, como también las posiciones excluyentes para ciertas identidades son producidas bajo los rótulos de "conocer y aprender."

Para que el currículum pueda ser pensado en su carácter generativo, debo recurrir a la noción de discurso, que la entiendo como la trama de relaciones de poder y conocimientos que van más allá, pero que incluyen el lenguaje hablado y escrito como también las imágenes usadas en espacios tanto populares como académicos. Weedon (1987, p. 108) explica de manera clara la definición de discurso según Foucault,

Los discursos, en el trabajo de Foucault, son formas de constituir conocimiento, junto con las prácticas sociales, formas de subjetividad y relaciones de poder las cuáles son inherentes a estas formas de conocer y las relaciones entre ellas. Los discursos son más que formas de pensar y producir significado. Ellos constituyen la "naturaleza" del cuerpo, de la mente consciente e inconsciente y de la vida emocional de los sujetos que busca gobernar. Ni el cuerpo, ni la mente, ni las emociones tienen significados fuera de su articulación discursiva, pero las formas en las que el discurso constituye las mentes y cuerpos de los individuos es siempre parte de una red más amplia de relaciones de poder, a menudo con bases institucionales.

Por ejemplo, el poder de los discursos de la "vulnerabilidad" y de las "culturas marginales", tan recurrentes para constituir identidades escolares en necesidad de estrategias compensatorias para la promoción de "buenos desempeños," instala, por lo menos, dos verdades. Por un lado, se hace real la idea de sujetos vulnerables, y como consecuencia reproduce el discurso de aquellos estudiantes no vulnerables, los que no necesitan ser reducidos a ninguna categoría. Por lo tanto, estos últimos quedan conformados como los y las estudiantes modelos (los que nos son vulnerables ni pertenecen a 
culturas marginales) en donde sus "logros" son atribuibles a valores culturales compartidos, como por ejemplo el apoyo de la familia y la exhibición de un fuerte componente ético y valórico. Estos discursos instalan una idea de que existe una normalidad en la escuela que tiene una clase social, tiene un color, tiene un género y tiene una sexualidad. Por lo tanto, no es sorpresa que los discursos para producir las identidades de los y las estudiantes nunca son neutrales, más bien están anclados y reflejan posiciones políticas, valores y prácticas sociales (HALL, 1990; WEEDON, 1987).

Por lo tanto, el currículum actúa como un mecanismo de referencia para explicar posiciones sociales y culturales de los sujetos. En tanto el currículum promueve la idea de que las identidades son naturalmente otorgadas (estudiantes pobres, vulnerables, los hombres, las mujeres, estudiantes con necesidades educativas especiales, peruano, chileno, etc.) - esto a través de no cuestionar su carácter histórico y discursivo--produce también que caracterizaciones de estudiantes, tales como, "resistentes al orden establecido" sean posibles y naturalizadas reproduciendo ideas y representaciones altamente dañinas. Estas, más que representaciones de una realidad, son el reflejo y efecto de las dinámicas de las relaciones de poder y de la repetida expresión y circulación irreflexiva de éstas en la escuela y en los discursos populares.

Ahora bien, la tendencia generalizada al desarrollar planes y programas escolares es pensar el conocimiento (entendido como los contenidos disciplinares) como neutral, intocable, inamovible. Esto es profundamente problemático porque una vez más se usa la neutralidad, en este caso, a través del currículum, para no complejizar aquello que se produce en la escuela. En este artículo, el conocimiento se entiende como una práctica (PINAR, 2000; SLAT'TERY, 2006; POPKEWITZ, 1997), por lo tanto, produce ideas, identidades e imaginarios acerca de lo que somos y de las posiciones sociales y culturales que ocupamos. El proponer conceptual y teóricamente al currículum como práctica de significación (PINAR et al., 2000; PARASKEVA, s/f.) ofrece la posibilidad de vincular sistemas de razonar dimensiones claves para la formulación, producción y circulación del conocimiento y las consecuencias que esto tiene en la producción de identidades de los sujetos. Varios autores contemporáneos que trabajan la idea del currículum entendido como una práctica de significación (DA SILVA, 2000; CHERRYHOLMES, 1999; PINAR, 2000; SLATTERY, 2006; BRITZMAN, 1991; LATHER, 1991, entre otros), proponen que existe la necesidad de movilizar el concepto de teoría curricular al de discursos del currículum. Esto, de manera reduccionista, plantea 
que "un discurso sobre el currículum, incluso cuando sólo pretende describirlo tal cual es, lo que hace es crear una idea particular de currículum. La supuesta descripción es, de hecho, una creación" (DA SILVA, 2001, p. 12). En este sentido, la noción de discurso curricular tiene que ver con que el currículum produzca y haga circular supuestos de realidades y nociones de sujeto, y que, por lo tanto, no se limita a reflejar o ser representación de una realidad, sino que es parte constitutiva de su creación. De esta manera, los críticos curriculares que trabajan en la perspectiva post-estructuralista coinciden en que las organizaciones, las instituciones, las categorías sociales, los conceptos, las identidades y sus relaciones son discursivas en su naturaleza. Por ende, el currículum "hace" y construye mundos más que representar verdades acerca de éste (PINAR et al., 2000; SLAT'TERY, 2006).

En este sentido, el currículum, a través de sus diferentes reformas, no es que haya "sido" algo que corresponda a una "realidad" que existía fuera del campo de significación curricular, sino que más bien el currículum es formado dentro de este campo. Por lo tanto, el currículum se ha formado en una trayectoria histórica en donde es posible rastrear los sistemas de ideas a los que hace referencia en tanto instalan modos de pensar, visiones del mundo particulares y distinciones conceptuales en la práctica escolar en general y en las asignaturas en particular. Por lo tanto, en este artículo el currículum se define como aquellas categorías del conocimiento formadas social e históricamente, que imprimen reglas por medio de las cuales las personas ven al mundo y se ven a sí mismas como miembros de ese mundo (POPKEWITZ, 1997). El estudiar el currículum escolar desde esta perspectiva ofrece una serie de posibilidades para la producción de políticas de producción de conocimiento escolar que den cuenta de problemáticas complejas dentro de la escuela.

\section{CIFRAS Y CONTEXTOS}

La producción de conocimiento escolar y las prácticas que se generan a partir de los sistemas aprendidos para razonar dimensiones tales como género, sexualidad, raza y clase en las escuelas chilenas produce la marginación de grupos, situación que queda expresada en los crecientes números de abuso, bullying y discriminación en las escuelas. Para enmarcar el problema, la idea no es poner atención a la segregación misma del sistema educativo chileno y abordar las diferencias desde la "brecha académica" entre distintos establecimientos, sino que es explorar cómo las formas de 
razonar sobre quiénes somos y las posiciones sociales y culturales que ocupamos en la sociedad son aprendidas/enseñadas en las trayectorias curriculares. Por lo tanto, la preocupación que lleva a plantear este trabajo tiene como fundamentos, al menos, dos grupos de datos relevantes. Primero, las estadísticas que indican altos porcentajes de estereotipos, prejuicio y discriminación existentes en el sistema escolar. Segundo, las políticas educativas que han emergido con el fin de regular y sancionar aquellos comportamientos que no promueven una convivencia sana y de calidad en los establecimientos educacionales. En referencia al primer grupo de antecedentes, las estadísticas de los últimos años en Chile, en relación a temas como género, sexualidad, VIH/sida, familia, entre otros, indican que hay una porción importante de nuestra población que discrimina (UNICEF, 2004, 2011; CEP, 2012; VIDAL et al., 2007). Por otro lado, estas cifras indican que los/las estudiantes, a medida que avanzan en la trayectoria escolar, van "convirtiéndose" en sujetos que reproducen ideas esencialistas en relación a género, sexualidad, raza y clase. Por discursos esencialistas aprendidos en la escuela se entiende aquellas prácticas discursivas que "tienden a establecer vínculos causales entre identidades y las experiencias sociales o comportamientos de las personas" (RASMUSSEN, 2006, p. 63). Junto con esta esencialización, la jerarquización de estas identidades esencializadas provoca que existan lugares epistémicos superiores y subordinados (no todos/ as tienen la autoridad para nombrar las identidades que se toleran y respetan) lo que tiene como efectos prácticas discriminatorias, de abuso físico, emocional y bullying. Profundizando más aún en las estadísticas, en la última encuesta UNICEF (2011) en donde se encuesta a niños y niñas, jóvenes de $7^{\circ}$ a $4^{\circ}$ año medio en relación a temas de discriminación y prejuicios, se concluye que los adultos son los que más discriminan en la escuela; el mayor grado de prejuicio se expresa hacia la homosexualidad y a personas con VIH/sida; se mantienen los prejuicios sobre género y etnia entre las encuestas de los años 2004 y 2011; y en general las bromas que hacen los/las estudiantes con más frecuencia son relativas a la diferencia de cualquier tipo. En el caso de esta encuesta es interesante resaltar que los y las estudiantes menores de 14 años se sienten más discriminados que los y las adolescentes, como también que un 49,9\% de los y las estudiantes de escuelas municipales se sienten discriminados permanentemente. Otro elemento a destacar es que frente a aseveraciones tales como "los hombres deben proteger a las mujeres, pues ellas son más débiles," un poco más del $80 \%$ está de acuerdo con esta aseveración, y 
nuevamente se destaca el alto porcentaje de participantes menores de 14 años respondiendo afirmativamente a este tipo de aseveraciones. Junto con este tipo de datos, la encuesta CEP (2012) que indaga por conceptos de mujer, familia y trabajo, también entrega información relevante para entender las formas de razonar la diferencia que se reproducen en la sociedad chilena. Por ejemplo, frente a la pregunta de quién debiera organizar ciertas actividades de la casa (ej. preparar la comida, lavar la ropa, cuidar enfermos, limpiar la casa, etc.) entre el $58 \%$ y $77 \%$ de los y las encuestadas, piensan que éstas debieran ser realizadas por mujeres. Vale considerar que esta encuesta considera como respondientes válidos a personas desde los 18 años de edad. Lo relevante de estas cifras es que este ordenamiento social y cultural se aprende en la escuela a través de diversos mecanismos, muchos de los cuáles han sido documentados y enfatizan la dimensión actitudinal frente a la diferencia, la importancia de la convivencia sana entre pares y relaciones interpersonales, entre otros (CASAS et al., 2008; FUNDACIÓN CHILE UNIDO, 2002; INFANTE; MATUS, 2009; MATUS; INFANTE, 2011; MINEDUC, 2007; SCHIAPPACASSE, 2008). Sin embargo, lo que me interesa relevar es el hecho de que es en la escuela en que se aprende a razonar la esencialización de las identidades, a ordenar las identidades en buenas/malas; mejores / peores o normales/raras, y también se aprende a privilegiar ciertas identidades por sobre otras. Si bien es cierto, muchos estudios destacan la influencia de los medios de comunicación, la publicidad, la familia y otras instituciones sociales en la producción de estas formaciones, lo que planteo es que hay que explorar en el conocimiento (currículum formal e informal) escolar porque si los y las estudiantes aprenden esta forma de razonar las identidades y sus relaciones, entonces es un tipo de conocimiento que se puede localizar y rastrear. Por tanto, el problema no se enfoca desde la forma tradicional de pensar cuáles son los/las estudiantes de diferentes contextos que están aprendiendo de manera diferente, en donde el supuesto es que los y las estudiantes de sectores más "vulnerables" no están expuestos a las mismas experiencias de aprendizajes, recursos, motivación por parte del o la profesora. Más bien, el problema es que todos y todas las estudiantes están aprendiendo formas que perpetúan sistemas de razonamiento naturalizadores de identidades, comunidades y relaciones. Los supuestos que sostienen que las prácticas y conocimiento discriminatorio son parte de una forma de conocer indica que, por lo tanto, se aprende (BRITZMAN, 1992) y que, como consecuencia, se puede desaprender o aprender de otra manera. 
Enreferencia al segundogrupodeargumentos para fundamentar la problemática, se encuentran las nuevas políticas educativas que buscan regular los abusos y prácticas violentas dentro de la escuela con leyes que instalan normas de sanción y castigo (LEY SOBRE VIOLENCIA ESCOLAR, 2011; LEY SOBRE CONVIVENCIA ESCOLAR, 2011). Estas formas de enfrentar los temas de abusos y discriminación, por lo menos, provocan preguntarse por el tipo de trabajo que debiera hacerse antes de llegar al tema de la sanción y castigo. El abordar estos problemas de bullying y discriminación con prácticas orientadas a la tolerancia y al respeto "por el/la diferente" no modifican estructuras del cómo se razona la diferencia. Más bien, la tolerancia es una forma de regular la aversión hacia esta diferencia y, por lo mismo, genera otros tipos de discriminación. Estas prácticas más bien instalan la producción de sujetos marcados para ser tolerados, lo que, para este artículo, es considerado como parte del problema, si la idea es construir sociedades más justas y democráticas. Los discursos de tolerancia, posicionan a los sujetos marcados como desviados, marginales o indeseables por lo que están en virtud de ser tolerados, lo que inevitablemente trae consigo el ejercicio de una superioridad, no problematizada, por parte de aquellos/as que son entrenados/ as para tolerar. Por ejemplo, Wendy Brown (2006) explica que los discursos de tolerancia promueven, simultáneamente, tres acciones: (1) intensifican los efectos reguladores de las identidades esencializadas; (2) ideológicamente, constituye una "diferencia" fuera de un sistema de subordinación o inequidad; y (3) fortalece la hegemonía de la identidad no marcada o dominante. Por ejemplo, cuando los/as estudiantes de educación básica son invitadas a tolerar la raza, etnia, cultura, religión, orientación sexual de otros/as, no hay ninguna sugerencia de cómo estas diferencias o identidades son producidas y circuladas, de cómo han sido histórica y culturalmente construidas y, menos aún, mostrar que ellas mismas son el efecto del poder y de la instalación y operación de las normas hegemónicas. Más bien, la diferencia misma es la que los/las estudiantes aprenden a tolerar. Las lógicas de sanción y castigo, más los discursos instalados de tolerancia y respeto por la "diferencia" que hoy ocupan buena parte de los discursos oficiales de los establecimientos educativos, promueven formas de organizar la diferencia y la normalidad que reproducen sistemas desiguales.

En el siguiente apartado quiero presentar como el uso de las "actitudes" se ha transformado en la forma en que el currículum consolida una forma de reproducir discursos de normalidad y diferencia en la escuela. 
En esta sección voy a explorar como opera la reinscripción del sentido común a través del uso del afecto en el currículum oficial en Chile. El uso de las actitudes en el currículum oficial convierte al afecto en público, común y obvio (STEWART, 2007). De esta manera, el afecto permite una reproducción espontánea de discursos culturalmente dominantes que normaliza cuerpos, relaciones y prácticas de reconocimiento, las que al mismo tiempo intensifican formas específicas de conocer y producir los mundos sociales y culturales propios y de los/as otros/as.

$\mathrm{El}$ afecto produce y refuerza significados particulares acerca del ser normal y ser distinta/o y remite a formas específicas de generar valor y premiación por el "buen comportamiento." De muchas maneras, el afecto es usado en el currículum para "gobernar a la distancia" (ROSE, 1999). Por lo tanto, el afecto es conceptualizado como esa fuerza que nos permite mapear tecnologías, conexiones, formas de razonar los cuerpos y las prácticas en relación con ideas específicas acerca de quienes debieran ser las y los estudiantes y como debieran comportarse. El afecto se moviliza a través de redes de relaciones, escenas, contingencias y emergencias, todas complejas. El afecto, como algo que ocurre, actúa como una fuerza que no tiene como localizarse y que no pertenece a nadie. Esto es sentido común (STEWART, 2007).

Hay una distinción conceptual importante y necesaria para referirnos al afecto. Afecto es muchas veces relacionado con el concepto de emoción. Para establecer una diferencia considero el trabajo de Massumi (2002, p. 27) que define afecto como un estado que "sigue diferentes lógicas y que pertenece a diferentes órdenes". Esto significa que afecto se distancia de las lecturas más psicológicas y de las teorías dominantes de la significación. Dicho de otra manera, la emoción es "leíble" y el afecto es disperso. Como Massumi (2002, p. 28) explica "Una emoción remite a un contenido subjetivo, la fijación sociolinguística de la calidad de una experiencia que forma el punto desde el cual se define lo personal. La emoción es intensidad calificada, lo convencional, el punto de consenso de la inserción de la intensidad en progresiones formadas semántica y semióticamente, son los circuitos de la acción-reacción narrable, es función y significado. Es intensidad apropiada y reconocida". Afecto, por el otro lado, nos habla de formas de conocer, pulsar, vivir, accionar la vida. Como Katheleen Stewart (2007, p. 4) comenta, 
[...] los afectos comunes son más atractivos que las ideologías, como también son más irritables, múltiples e impredecibles que los significados simbólicos. Los afectos comunes no son el tipo de objeto analítico que puede ser dispuesto en un plano de análisis singular y estático y tampoco se prestan para un paralelismo perfecto entre el sujeto analítico, el concepto y el mundo. Al contrario, los afectos son un problema o una cuestión emergente en escenas dispares, formas y registros inconmesurables; un enredo de conexiones posibles.

Los afectos por lo tanto, son expresiones performadas como intensidades y variaciones de los mundos culturales y sociales. $\mathrm{Ni}$ afecto ni afección denotan un sentimiento personal. Como Massumi (2002, p. xvi) explica "es una intensidad pre-personal que corresponde al tránsito de un estado de experiencia del cuerpo a otro y que implica el aumento o disminución de la capacidad del cuerpo para actuar".

Habiendo explicitado conceptualmente la distinción entre emoción y afecto, me gustaría mostrar lo que se expone en el currículum oficial como actitudes y las razones que se plantean para sus usos. Las Bases Curriculares ${ }^{1}$ es el documento que especifica los objetivos de aprendizaje para cada asignatura escolar en Chile. Las Bases Curriculares es un documento que fue oficializado en el año 2012 y se constituye en la reforma educativa instalada por el gobierno actual en donde se indica expresamente el valor y posición que tienen las actitudes para el éxito de los aprendizajes. Las Bases Curriculares detallan un conjunto de actitudes específicas que se espera desarrollar en cada asignatura y las define de la siguiente manera,

Se espera que, desde los primeros niveles, los alumnos hagan propias estas actitudes, que se aprenden e interiorizan mediante un proceso permanente e intencionado, en el cual es indispensable la reiteración de experiencias similares en el tiempo. El aprendizaje de actitudes no debe limitarse solo a la enseñanza en el aula, sino que debe proyectarse socialmente y ojalá involucrar a la familia. (BASES CURRICULARES, 2012, s/n).

Para hacer efectiva esta definición se ha diseñado un set de actitudes por asignatura, lo que indica que cada conocimiento o contenido está relacionado con formas específicas de usar el afecto como asegurador de la concreción de otros conocimientos como son Historia, Lenguaje, etc. Es así como se indica que las actitudes,

son disposiciones aprendidas para responder, de un modo favorable o no favorable, frente a objetos, ideas o personas; incluyen componentes afectivos, cognitivos y valorativos, que inclinan a las personas hacia determinados tipos de conductas o acciones. Las actitudes cobran gran importancia en el ámbito educativo, porque trascienden la dimensión cognitiva y se relacionan con lo afectivo. El éxito de los aprendizajes depende en gran medida de las actitudes y disposiciones de los estudiantes. Por otra parte, un desarrollo integral de la persona implica, necesariamente, el considerar los ámbitos personal, social y ético en el aprendizaje. (BASES CURRICULARES, 2012, s/n). 
Quiero presentar el caso particular de 4to año básico y las asignaturas de Historia y Ciencias Sociales y Ciencias Naturales con el fin de ilustrar los usos que se hace del afecto. He escogido estas dos asignaturas porque me parece que son dos áreas del conocimiento que son "evidentes" en tanto organizan sus conocimientos alrededor de construcciones esencialistas. Por ejemplo, las construcciones del "Nosotros vs. Ellos," tan evidentes en la forma en que aprendemos Historia, ha llevado a pensar que la diferencia se constituye tanto en relación a una idea inamovible de normalidad como también a través de las prácticas esencialistas y homogeneizantes inherentes al concepto de cultura. Estas ideas se han mantenido de manera romantizada en el currículum y textos escolares, a pesar de que han sido ampliamente problematizadas en áreas como la antropología y la teoría critica (MAHALINGAM; MCCARTHY, 2000). Para muchos críticos las ideas de multiculturalidad son usadas como una herramienta para esencializar culturas y también como una extensión de lo denominado educación cívica, que sería algo así como que "existen otras culturas en el mundo y que nosotros/as debiéramos respetar." Dicho de otra manera, las experiencias de marginalización encarnadas en las identidades aprendidas en asignaturas como Historia, produce la reiteración de una concepción de cultura entendida como una colección de objetos y prácticas folclóricas y estéticas que remiten a grupos específicos. Esta forma conveniente de homogeneizar en unidades discretas identidades y comunidades tiene efectos críticos en cómo los y las estudiantes se piensan a ellas mismas y a otros.

En el caso de Ciencias Naturales, las nociones de biología y naturaleza construyen sistemas de razonar que permean las prácticas escolares. Por ejemplo, los ordenamientos temporales en las distribuciones de los/las estudiantes en el sistema escolar obedece a la idea de que hay una realidad biológica que define las capacidades cognitivas, actitudinales, emocionales y los desarrollos fisiológicos (SEATON, 2012). Por otro lado, la construcción del cuerpo y su disciplinamiento (WAGENER, 1998) ha sido usado como "evidencia" o "idea esencial" (SEATON, 2012) para todo tipo de proliferación de ideologías. El cuerpo se construye en el currículum escolar como materia "natural" e independiente de las influencias sociales. Aún cuando el binomio cuerpo-verdad es arbitrario, es a partir de estas construcciones que es posible crear ideas tales como la rareza, deformidad y déficit de los cuerpos. Más aún, la asignación de atributos a los cuerpos es parte constitutiva de ideas esencialistas de género, tan expresamente aprendidas en la escuela a través de la noción de que el cuerpo ofrece evidencia biológica para construir las diferencias de comportamiento 
de los géneros. Como síntesis, la dialéctica entre biología y naturaleza, provista en las Ciencias Naturales, más la noción de cultura entregada por la disciplina de Historia y Ciencias Sociales, me proporcionan dos lugares claves para entender las conexiones esencialistas entre el mundo de lo social y lo natural desde el que se construyen ideas de quiénes somos y quiénes son los "otros" y de las complejas relaciones y atribuciones, no sólo en el sentido de la producción semiótica de un binarismo sino que en la construcción necesaria de relaciones de "reciprocidad" que orientan nuestras narrativas de vida.

A continuación, presento un listado con las actitudes que se han definido en el currículum oficial para las asignaturas de Historia y Ciencias Sociales y Ciencias Naturales para 4to ano básico con el fin de mostrar cómo funcionan estas ideas en la producción de la normalidad y la diferencia.

Actitudes para la asignatura de Historia y Ciencias Sociales de 4to año básico:

a. Demostrar valoración por la vida en sociedad para el desarrollo y el crecimiento de la persona.

b. Demostrarvaloración porlademocracia, reconociendo su importancia para la convivencia y el resguardo de derechos. c. Comportarse y actuar en la vida cotidiana según principios y virtudes ciudadanas.

d. Establecer lazos de pertenencia con su entorno social y natural a partir del conocimiento, la valoración y la reflexión sobre su historia personal, su comunidad y el país.

e. $\quad$ Participar solidaria y responsablemente en las actividades y proyectos del establecimiento y del espacio comunitario, demostrando espíritu emprendedor.

$f$. Respetar y defender la igualdad de derechos esenciales de todas las personas, sin distinción de sexo, edad, condición física, etnia, religión o situación económica.

g. Respetar y defender la igualdad de derechos entre hombres y mujeres y apreciar la importancia de desarrollar relaciones que potencien su participación equitativa en la vida económica, familiar, social y cultural.

h. Reconocer la importancia y la dignidad de todos los trabajos, valorando y respetando a las personas que los realizan. i. Trabajar en forma rigurosa y perseverante, con espíritu emprendedor y con una disposición positiva a la crítica y la autocrítica. 
Actitudes para la asignatura de Ciencias Naturales de 4to año básico:

a. Demostrar curiosidad e interés por conocer seres vivos, objetos y/o eventos que conforman el entorno natural. b. Manifestar un estilo de trabajo riguroso, honesto y perseverante para lograr los aprendizajes de la asignatura.

c. Reconocer la importancia del entorno natural y sus recursos, desarrollando conductas de cuidado y protección del ambiente. d. Asumir responsabilidades e interactuar en forma colaborativa y flexible en los trabajos en equipo, aportando y enriqueciendo el trabajo común.

e. Manifestar compromiso con un estilo de vida saludable por medio del desarrollo físico y el autocuidado.

f. Reconocer la importancia de seguir normas y procedimientos que resguarden y promuevan la seguridad personal y colectiva.

He subrayado algunas de las actitudes que se presentan en el documento oficial con el fin de organizar la discusión en relación a que es lo que "hace" esta forma de promover aprendizajes a través del afecto. Para comenzar, me gustaría indicar que una de las primeras distinciones necesarias de hacer es que algunos teóricos promueven que estas actitudes, como por ejemplo, la del "autocuidado" (en el listado de actitudes de la asignatura de Ciencias Naturales) es una capacidad afectiva y cognitiva por lo tanto puede ser cultivada para aumentar las habilidades morales y promover las relaciones éticas entre personas que pertenecen a distintos territorios, culturas y grupos. Pero me gustaría intencionar la discusión de que también se puede decir que "autocuidado" es una relación social o un producto de la circulación de otros conocimientos (AHMED, 2004; 2010; BERLANT, 2004). Si esta discusión se centra en la primera versión de entender las actitudes, tendríamos problemas tales como que "autocuidado" se transforma en una propiedad, como algo que alguien tiene, y que puede ser traspasado, aprendido, evaluado, monitoreado (lo que sería en el caso de la propuesta curricular en cuestión). Por lo tanto, no es que la idea de "autocuidado" reside "dentro del individuo" sino que es un "espacio mediado" (BOLER, 1999). Esta autora plantea que las "emociones son un medio, un espacio en el cual las diferencias y las éticas son comunicadas, negociadas y toman formas" (BOLER, 1999, p. 21). Desde esta perspectiva, el "autocuidado" es entendido de una manera productiva (que produce prácticas, relaciones y subjetividades), como una inversión para la producción de normas sociales y relaciones de poder. 
El "autocuidado" puede ser entendido como una tecnología afectiva de "preocuparse por sí mismo/a" y que es movilizada para mantener un orden desde la economía (formas de regular los excesos en la población) y el orden social. El "autocuidado" se transforma en una tecnología de regulación, ya que produce y diferencia sujetos afectivos y los medios para gobernarlos. En este caso "las [actitudes] nos pueden atar a las mismísimas condiciones de nuestra subordinación" (AHMED, 2004, p. 12). El "autocuidado" puede ser visto como un instinto individual, que cuando es cultivado apropiadamente se traduce en una competencia colectiva que produce valor económico, cultural y político (PEDWELL, 2012). En el caso del autocuidado como una actitud a desarrollar, está a la superficie de una forma de afectar al sujeto para actuar de una manera en particular. El afecto da significado a una actitud y actúa como una medida objetiva de situaciones particulares vividas en contextos particulares. Más importante aún, cuando el afecto es usado para generar prácticas sociales y culturales específicas, estas no son relevantes excepto cuando están en relación con el desarrollo de una economía afectiva. Para definir como una economía afectiva opera, necesariamente hay que referirse a aquellos "valores afectivos [que] les corresponde a una imagen que está en circulación (WISSINGER, 2007, p. 239). Esto sin duda que remite al contexto político chileno en donde los supuestos neoliberales informan las reformas curriculares expuestas. Esta cadena de afectos presentados en estas dos asignaturas actúan como vigilantes de la moralidad de los y las estudiantes y de sus familias. El afecto expresado en las actitudes de las asignaturas estructura perfiles sociales y culturales específicos que son centrales para las consecuentes explicaciones reduccionistas del valor del vínculo de la familia y la escuela, de cómo la vulnerabilidad es un problema para el éxito escolar, etc.

Creo que es importante intencionar la exploración de como el "sujeto afectivo" es construido en redes discursivas normativas y dominantes acerca de cómo se aprende Historia, Ciencias Sociales y Ciencias Naturales, particularmente, porque el contexto de las políticas educativas actual tiende a generar políticas punitivas para el trabajo escolar. Estas actitudes presentadas reflejan la forma instrumental en que se enmarcan los temas sociales y culturales en contraste con cualquier reflexión de como las construcciones de raza, clase, género, etc. podrían informar las causas de estas situaciones. Por lo tanto, el uso del afecto para incitar el aprendizaje de estas actitudes actúa como una demanda por asimilación de ciertas subjetividades y comportamientos, atribuyendo estas a contenidos curriculares específicos (Historia, Ciencias Sociales 
y Ciencias Naturales). De esta manera, las actitudes son sentidas y vividas como verdades. Estas actitudes imponen en los y las profesoras y estudiantes formas irreflexivas de instalar conexiones entre redes homogéneas de afectos, deseos y posibilidades. Dicho de otra manera, se producen unos circuitos de performances corporales, tales que "las actitudes correctas se relacionan con comportamientos correctos, los comportamientos correctos aseguran aprendizajes eficaces del contenido disciplinar, los aprendizajes óptimos aseguran éxito escolar, así estos logros son reconocidos como recompensas sociales y culturales." Es así como el afecto se convierte en este tracing abstracto detrás de lo que reconocemos como aprendizaje dentro de las experiencias curriculares.

El currículum entendido como productor de subjetividades a través del uso del afecto nos abre una posibilidad de problematización que nos vincula a preguntas más relevantes que tienen que ver con cómo la política económica (neoliberalismo) requiere que el comportamiento de los sujetos sea educado y encauzado en la escuela. Sin duda alguna que la sofisticación con que el poder viaja demanda de nuestra creatividad para poder acceder a nuevas formas de teorizar nuestras trayectorias curriculares y sus efectos.

\section{REFERENCIAS}

AHMED, Sara. The promise of Happiness. Durham and London: Duke University Press, 2010. AHMED, Sara. The Cultural Politics of Emotions. New York: Routledge, 2004.

AOKI, T. Legitimating lived currículum: Toward a curricular landscape of multiplicity, 1993. Journal of Currículum and Supervision, Ohio State University Press, n. 8, v. 3, p. 255-168. BAEZ, B. The Study of Diversity: "the knowledge of difference" and the limits of science. The Journal of Higher Education, Ohio State University Press, v. 75, n. 3, May/June 2004, p. 285-306.

BACCHI, C.L. Women, Policy, and Politics. The Construction of Policy Problems. London: Sage, 1999.

BERLANT, L. Compassion: the Culture and Politics of an Emotion. New York: Routledge, 2004. BOLER, M. Feeling Power: Emotions and Education. London: Routledge, 1999.

BRITZMAN, D. Practice makes practice. A critical study of learning to teach. Albany, NY: State University of New York Press, 1991.

BROWN, W. Regulating Aversion. Tolerance in the Age of Identity and Empire. Princeton and Oxford: Princeton University Press, 2006.

CASAS, L; AHUMADA, C; RAMOS, L; GUAJARDO, A; CONTRERAS, D. La Convivencia Escolar, Componente Indispensable del Derecho a la Educación. 2008. Recuperado el 11 de Enero de 2012 del sitio web de la UNICEF, en < http:/ / www.unicef.cl/ unicef/public/archivos_documento/285/WD_11_Reglamentos_Convivencia.pdf>. 
CENTRO DE ESTUdiOS PUBLICOS. Encuesta de Opinion Publica. 2012, n. 66, en <http://www.cep.cl/abril2012.pdf>. March, 2014.

CHERRYHOLMES, C. Poder y Crítica: Investigaciones Postestructurales en Educación. Barcelona: Ediciones Pomare-Corredor, 1999.

EIDELMAN, S.; CRANDALL, C; GOODMAN, J.; BLANCHAR, J. Low effort thought promotes political conservatism. Personality and Social Psychology Bulletin, Los Angeles, Estados Unidos, v. 38, n. 6, p. 808-820, 2012.

FUNDACIÓN CHILE UNIDO. Bases para una Política de Educación Sexual. Recuperado el 11 de Enero de 2012 del Portal de la Familia, de < http://www.portaldafamilia. org/artigos/baseseducsex.pdf>. Marzo, 2014.

HALL, S. Cultural Identity and diaspora. In: RUTHERFORD, J. (Ed.). Identity: Community, Culture, Difference. London: Lawrence and Wishart, 1990. p. 222-239.

HENRY, M.; LINGARD, B.; RIZVI, F; TAYLOR, S. The OECD, Globalization and Education Policy. Oxford: Pergamon, 2001.

HUEBNER, D. Currículum as the concern for man's temporality. In: PINAR, W. (Ed.). In currículum theorizing: The reconceptualists. Berkeley, CA: Mc. Cutchan, 1975. p. 237-249.

INFANTE, M.; MATUS, C. Policies and Practices on Diversity: Possibilities to Reimagine New Discourses. Disability and Society, v. 24, n. 4, 2009. p. 437-445.

JARDINE, D. Reflections on education, hermeneutics, and ambiguity. In: PINAR, W.; REYNOLDS, W. (Eds.). Understanding currículum as phenomenological and deconstructed text. New York: Teachers College Press, 1992. p. 116-127.

LATHER, P. Getting Smart: Feminist Research and Pedagogy with/in the Postmodern. London, England: Routledge, 1991.

MACRINE, S. L. (Ed.) Inclusive Schooling, en David Gabbard: Knowledge and Power in the Global Economy. The effects of school reform in a neoliberal/neoconservative age. New York and London: Lawrence Erlbaum Associates, 2008. p. 449-461.

MAHALINGAM, R; MCCARTHY, C. Multicultural Currículum: New Directions for Social Theory, Practice, and Policy. New York: Routledge, 2000.

MASSUMI, B. Parables of the Virtual. Movement, Affect, Sensation. Durham \& London: Duke University Press, 2002.

MATUS, C; INFANTE, M. Undoing Diversity: Knowledge and Neoliberal Discourses in Colleges of Education, Discourse. Studies in Cultural Politics of Education, Australia, v. 32, n. 3, p. 293-307, 2011.

MINEDUC. Bases Curriculares. 2012. En: <http://www.mineduc.cl/index5_int.php? id_portal=47\&id_contenido=17116\&id_seccion=3264\&c=10>. Marzo, 2014.

MINEDUC. Ley sobre Convivencia y Violencia Escolar. 2011. En: < http://www.mineduc.cl/ usuarios/convivencia_escolar/doc/201109221119290.ley_violencia_escolar.pdf>. Marzo, 2014.

MINEDUC. Guía Introductoria. Respuestas Educativas a la Diversidad y a las Necesidades Educativas Especiales. Recuperado el 17 de Enero de 2012 del sitio web del MINEDUC, de < http://www.mineduc.cl/biblio/documento/200809221559390.0GuIaInt roductoria.pdf $>$. Marzo, 2014.

MINEDUC. Política Nacional de Educación Especial: nuestro compromiso con la diversidad. Santiago: Ministerio de Educación, 2005.

OEA. Convención Interamericana para la eliminación de todas las formas de discriminación contra las personas con discapacidad. Washington DC, USA, 1998. 
OREALC/UNESCO. Temario abierto sobre educación inclusiva. Materiales de Apoyo para responsables de Políticas Educativas. Santiago: Oficina Regional de Educación para América Latina y el Caribe, 2004.

ORGANIZATION FOR ECONOMIC COOPERATION AND DEVELOPMENT (OECD), Centre for Educational Research and Innovation (CERI). Retrieved July 20, 2009, from < http://www.oecd.org/dataoecd/38/18/38446790.pdf>.

PARASKEVA, J. (s/f). El currículo como práctica de significaciones. Traducción de Pablo Manzano. Recuperado el 2 de marzo de 2011 desde <www.quadernsdigitals.net>.

PEDWELL, C. Economies of Empathy: Obama, neoliberalism, and social justice. Environment and Planning D: Society and Space, v. 30, 2012, p. 280-297.

PINAR, W. et al. Understanding Currículum. An Introduction to the Study of Historical and Contemporary Currículum Discourses. New York: Peter Lang, 2000.

PINAR, W.; REYNOLDS, W.; SLATTERY, P.; TAUBMAN, P. Understanding currículum as a phenomenological text. In: PINAR, W; Reynolds, M; Slattery, P; TAUBMAN, P; M. (Eds.). Understanding Currículum. New York: Peter Lang, 2000. p. 404-449.

POPKEWITZ, T; BRENNAN, M. Foucault's Challenge: Discourse, Knowledge, and Power in Education. New York: Teachers College Press, 1997.

RASMUSSEN, M. L. Becoming Subjects: Sexualities and Secondary Schooling. New York: Routledge, 2006.

ROSE, N. Governing the Soul. The Shaping of the Private Self. Second Edition. London: Free Association Books, 1989/1999.

SCHIAPPACASSE, P. Segregación residencial y nichos étnicos de los inmigrantes internacionales en el Área Metropolitana de Santiago. Revista Geografía Norte Grande, n. 39, 2008. p. 21-38.

SEATON, E. Biology/Nature en Keywords in Youth Studies. LESKO, Nancy; TALBURT, Susan (eds.). Tracing affects, movements, knowledges. New York and London: Routledge, 2012. p. 24-29.

SLATTERY, P. Currículum Development in the Postmodern Era. New York: Routledge, 2006. STEWART, K. Ordinary Affects. Durham e London: Duke University Press, 2007.

DA SILVA, T. Espacios de Identidad. Barcelona: Ediciones Octaedro, 2001.

UNESCO. La Discriminación y el Pluralismo Cultural en la Escuela. Casos de Brasil, Chile, Colombia, México y Perú. Recuperado el 11 de Enero del $2012<$ http://unesdoc. unesco.org/images/0014/001459/145989s.pdf>.

UNESCO. Guidelines for inclusion: ensuring access to education for all. Paris: UNESCO, 2005.

UNESCO. Declaración de Salamanca y Marco de Acción sobre Necesidades Educativas Especiales. Conferencia Mundial sobre Necesidades Educativas Especiales: Acceso y Calidad. Salamanca, 1994.

UNICEF. Encuesta "La voz de niños, ninas y adolescentes". 2011. Recuperado en marzo del 2014 <http://www.unicef.cl/pdf/PPTLaVozDiscriminacion2011.pdf>.

UNICEF (2004). Los prejuicios de los niños, ninas y adolescentes. <http://www. unicef.cl/pdf/PPTlosprejuiciosdeninosninasyadolescentes2004.pdf $>$.

VIDAL, F.; CONCHA, R.; PALMA, I.; BARRIENTOS, J. et al. Sexualidad, Género y VIH/SIDA: ¿Qué piensan los futuros docentes chilenos/as? [Sexuality, Gender and HIV/ AIDS: What do future Chilean teachers think?] MINSAL, Proyecto Transversal. Fondo global para la lucha contra el sida, la tuberculosis y la malaria, Santiago, Chile, 2007. 
WAGNER, J. R. The construction of the body through sex education. En: Thomas Popkewitz \& Marie Brennan (ed.). Foucault's Challenge: discourse, knowledge, and power in education. New York and London: Teachers College Press, 1998. p. 144-169.

WEEDON, C. Feminist practice and poststructuralist theory. New York: Basil Blackwell, 1987. WISSINGER, E. Always on Display: Affective Production in the Modeling Industry. In: CLOUGH, P. T.; HALLEY, J. (Eds.) The Affective Turn. Theorizing the Social. Durham \& London: Duke University Press, 2007. p. 231-260.

NOTA

${ }^{1}$ A partir del año 2012, el Ministerio de Educación en Chile estableció un nuevo cambio curricular en el marco de la Ley General de Educación. De esta forma, en la actualidad se opera con tres documentos oficiales, a saber, las Bases Curriculares se implementaron a partir del 2012 de 1ero a 6to básico; el Ajuste Curricular del 2009 que opera de 7 mo básico a 2do Medio; y el Marco Curricular que regula desde 3ero a 4to medio.

Recibido: $19 / 05 / 2015$

Aprobado: 06/11/2015

Correo postal:

Vicuña Mackenna 4860, Comuna Macul Santiago | Chile C.P. 7820436 